\title{
Strength and deformation behaviors of methane hydrate- bearing marine sediments in the South China Sea during depressurization
}

Tingting Luo ${ }^{1 *}$, Tao Han ${ }^{1}$, Madhusudhan B.N² , Xiaodong Zhao ${ }^{1}$, Di Zou' ${ }^{1}$, Yongchen Song ${ }^{3}$

${ }^{1}$ State Key Laboratory for Geomechanics and Deep Underground Engineering, School of Mechanics and Civil Engineering, China University of Mining and Technology, Xuzhou 221116, P.R. China

2 Faculty of Engineering and the Environment, University of Southampton, Southampton SO16 7QF, U.K

${ }^{3}$ Key Laboratory of Ocean Energy Utilization and Energy Conservation of Ministry of Education, Dalian University of Technology, Dalian 116024, P.R. China

${ }^{*}$ Corresponding author: T. Luo (kdltt@cumt.edu.cn)
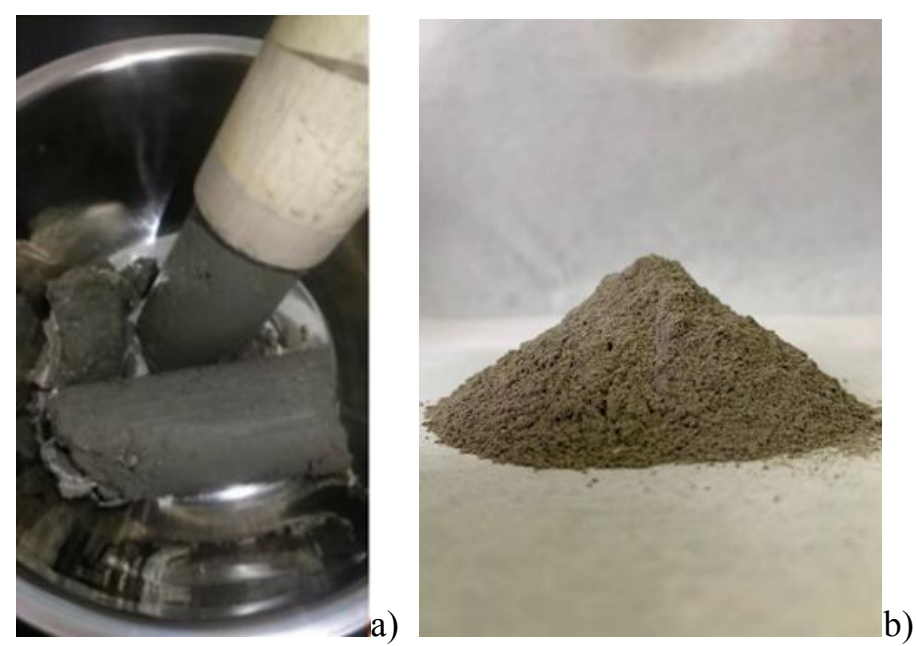

Figure S1 Marine sediments from the South China Sea: a) Global appearance; b) Accumulation 


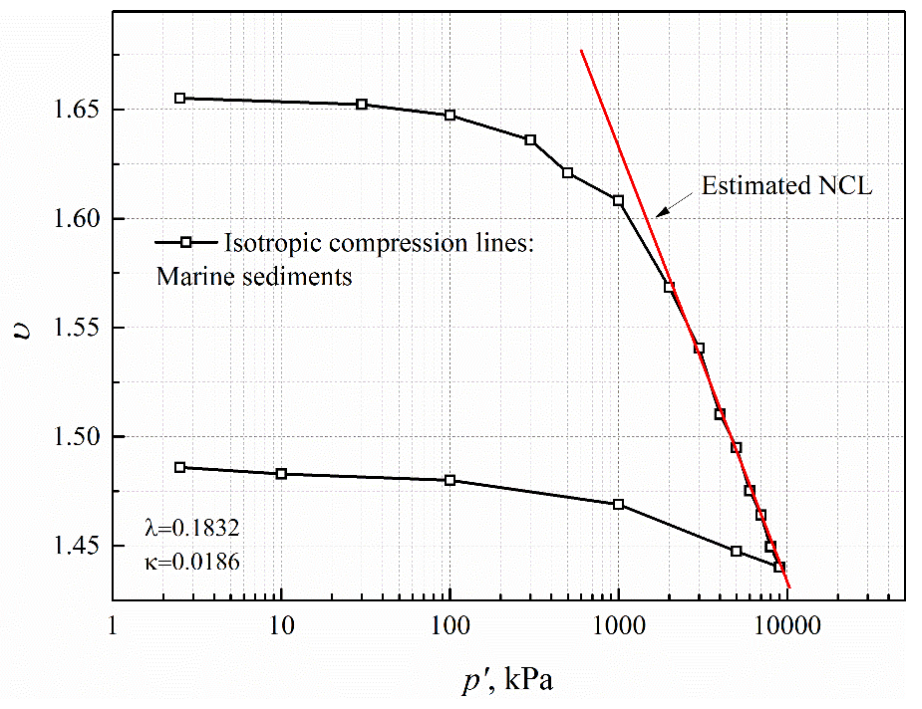

Figure S2 Isotropic compression behaviors of marine sediments

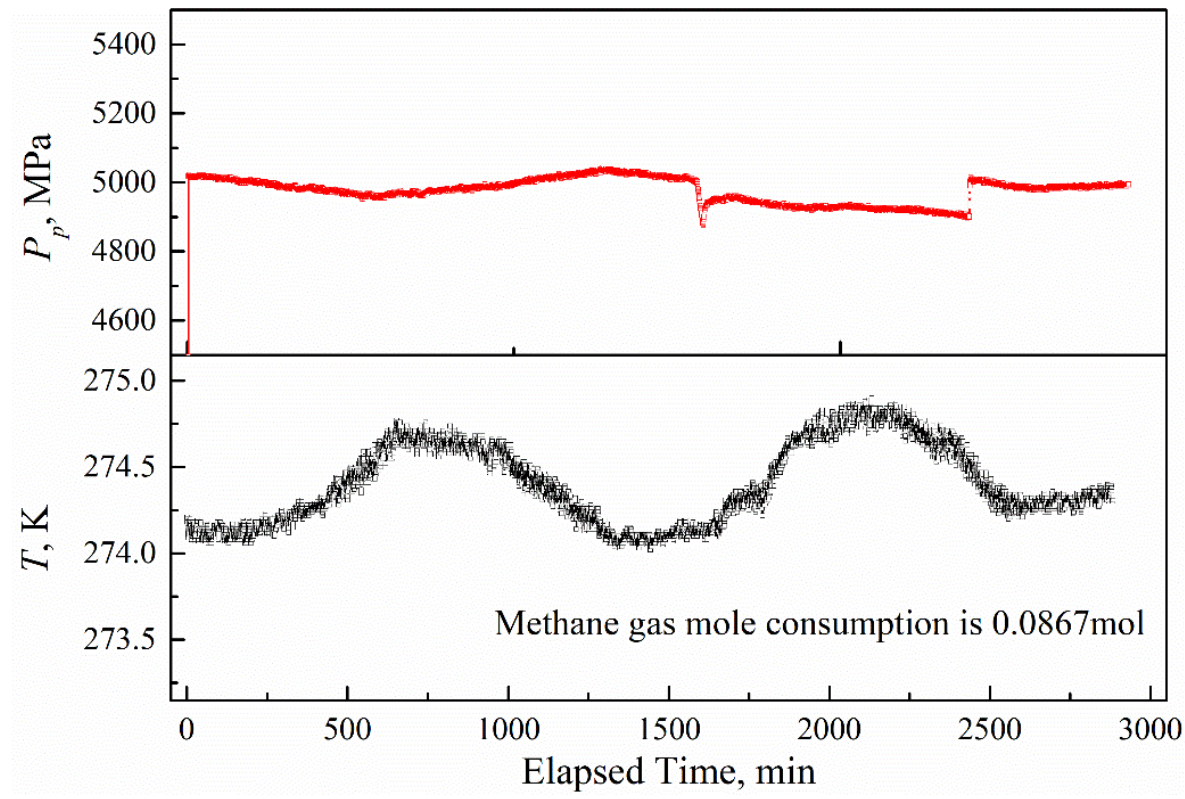

Figure S3 The pore pressure and temperature of the hydrate-bearing marine sediments in CaseED-4 during hydrate formation 


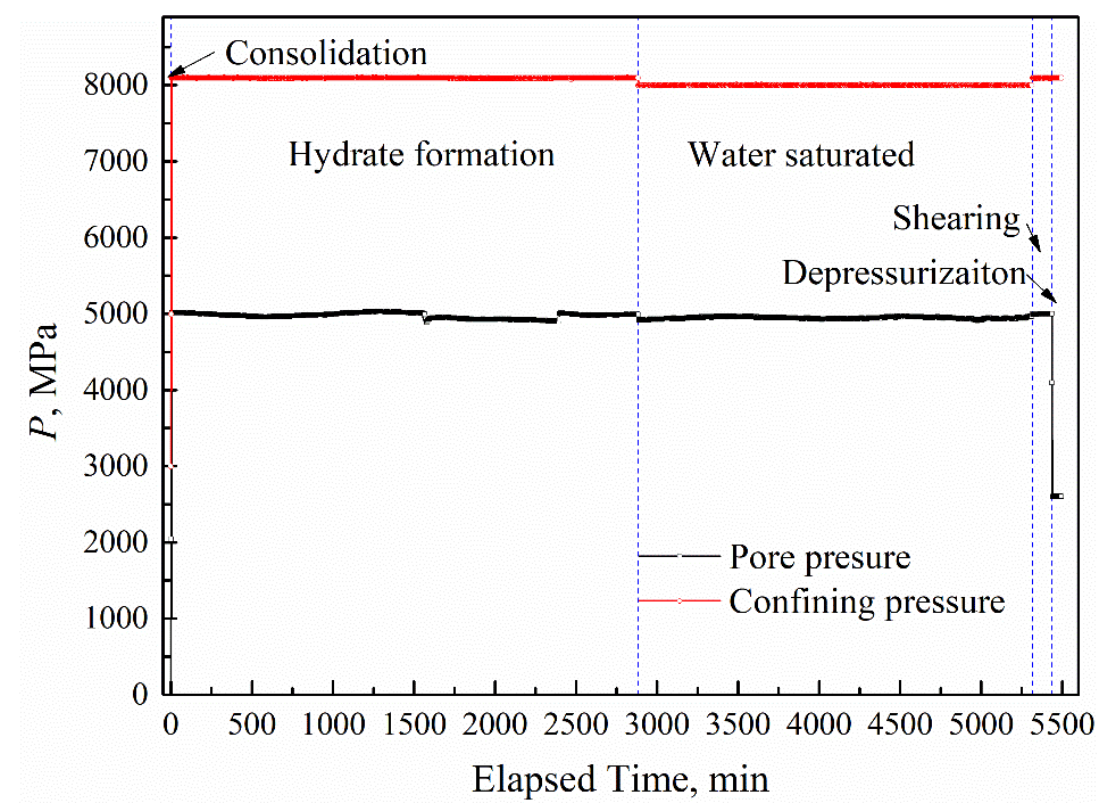

Figure S4 The pore pressure and confining pressure of the hydrate-bearing marine sediments in Case-ED-4 during consolidation, hydrate formation, water saturated, shearing and depressurization

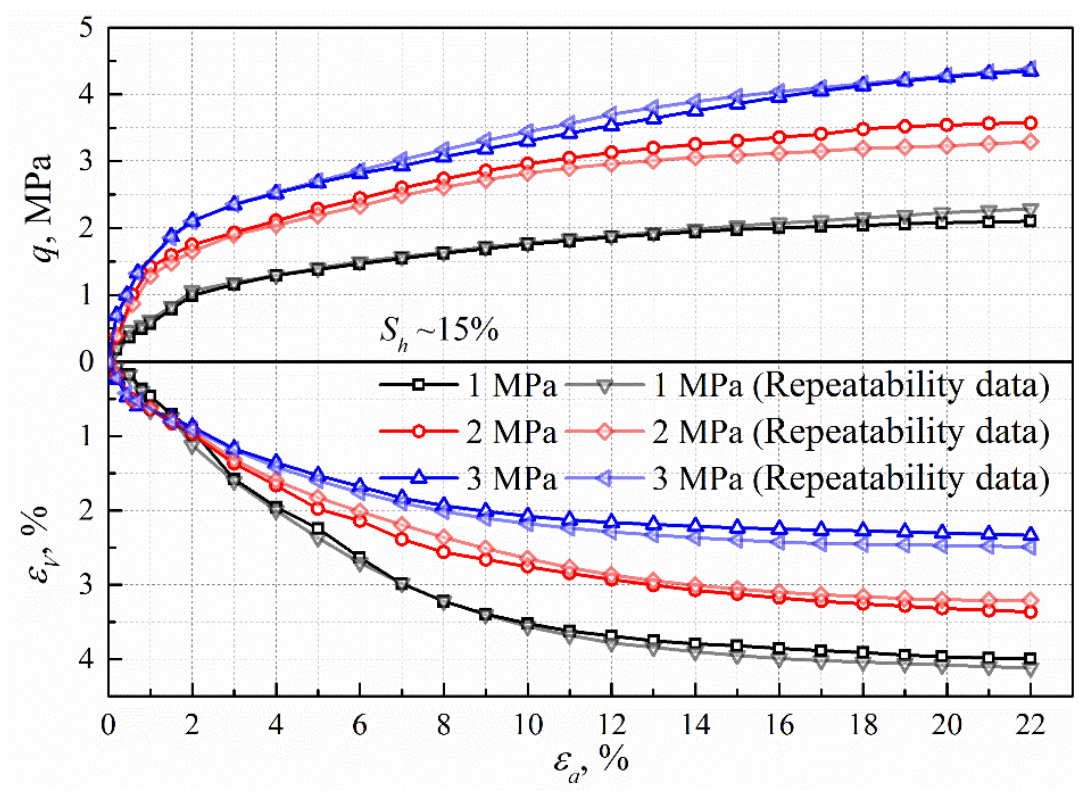

Figure S5 Stress-strain and volumetric strain behaviors of marine sediments with $15 \%$ hydrate saturation (with the repeatability data) 

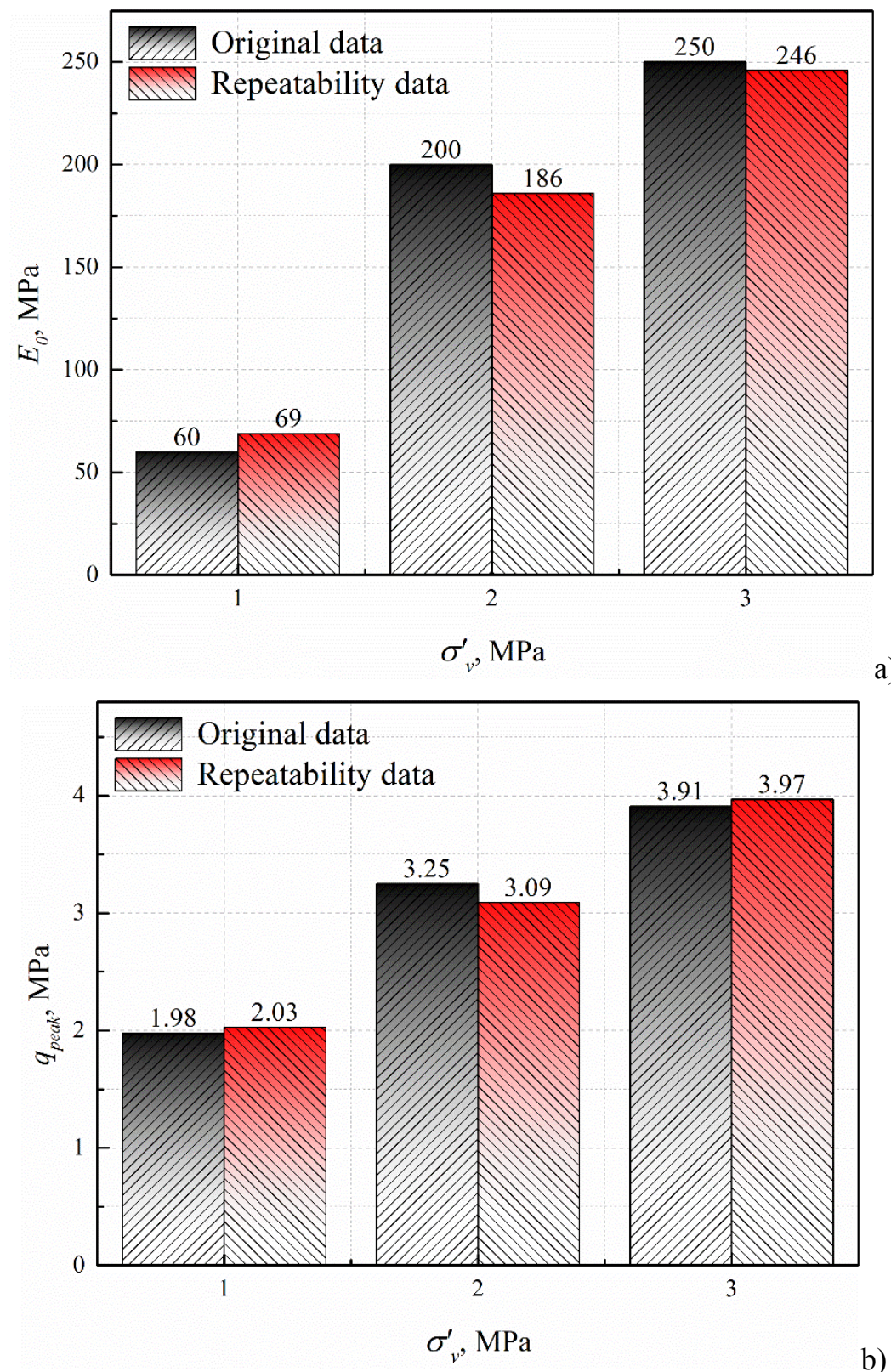

a)

b)

Figure S6 Parameters of marine sediments with different hydrate saturations: a) Elastic modulus; b) Peak strength (with the repeatability data) 